\title{
Apoptosis induction of human leukemia cells by Streptomyces sp. SY-103 metabolites through activation of caspase-3 and inactivation of Akt
}

\author{
SEONG-YUN JEONG ${ }^{1 *}$, MIN HO HAN ${ }^{2 *}$, CHENG-YUN JIN ${ }^{2}$, GI-YOUNG KIM ${ }^{3}$, \\ BYUNG TAE CHOI ${ }^{4}$, TAEK-JEONG NAM ${ }^{5}$, SE-KWON KIM ${ }^{6}$ and YUNG HYUN CHOI ${ }^{2}$
}

\begin{abstract}
${ }^{1}$ Department of Medical Life Science, Catholic University of Daegu, Daegu 712-702; ${ }^{2}$ Department of Biomaterial Control (BK21 program), Dongeui University Graduate School, Department of Biochemistry, Dongeui University College of Oriental Medicine, Busan 614-714; ${ }^{3}$ Faculty of Applied Marine Science, Cheju National University, Jeju Special Self-Governing Province 690-756; ${ }^{4}$ Department of Anatomy, Graduate School of Oriental Medicine, Pusan National University, Busan 609-735; ${ }^{5}$ Faculty of Food Science and Biotechnology; ${ }^{6}$ Department of Chemistry and Marine Bioprocess Research Center, Pukyong National University, Busan 608-737, Republic of Korea
\end{abstract}

Received August 14, 2009; Accepted October 12, 2009

DOI: 10.3892/ijmm_00000310

\begin{abstract}
We isolated 23 marine actinomycetes from seawater samples. Of these, strain SY-103 exhibited the strongest cytotoxic activity on human leukemic cell lines. Biochemical tests and 16S rDNA sequencing of this strain allowed us to identify SY-103 as a strain of the genus Streptomyces. In the present study, the pure cytotoxic compound (PCC) from Streptomyces sp. SY-103 metabolites was purified by reversephase HPLC and the biochemical mechanisms of PCCinduced apoptosis in cultured human leukemic cell lines were investigated. The exposure of cells to PCC resulted in growth inhibition and induction of apoptosis, which was associated with the proteolytic activation of caspase- 3 and down-regulation of anti-apoptotic Bcl-2 protein. However, PCC-induced caspase- 3 activation and apoptosis were significantly attenuated in Bcl-2 overexpressing U937 cells. z-DEVD-fmk, a caspase-3 specific inhibitor, blocked caspase- 3 activation and increased the survival rate of PCC-treated U937 cells. The activity of Akt was also inhibited in PCC-treated cells, and phosphatidylinositol 3-kinase (PI3K)/Akt inhibitor, LY294002, sensitized the cells to PCC-induced apoptosis indicating that the down-regulation of the Akt signaling pathway plays a key role in PCC-induced apoptosis. Our findings imply that some of the biological functions of Bcl-2 and PI3K/Akt are attributed to their ability to
\end{abstract}

Correspondence to: Dr Yung Hyun Choi, Department of Biochemistry, Dongeui University College of Oriental Medicine, Yangjung-Dong, Busanjin-Gu, Busan 614-714, Republic of Korea E-mail: choiyh@deu.ac.kr

*Contributed equally

Key words: Streptomyces sp. SY-103 metabolites, apoptosis, Bcl-2, caspase-3, PI3K/Akt inhibit PCC-induced apoptosis; therefore, it is suggested that this compound is a promising anti-cancer agent for leukemia cells.

\section{Introduction}

Actinomycetes are considered a pre-eminent source of bioactive natural products. They are responsible for the production of about half of the discovered bioactive secondary metabolites (1), including anti-tumor agents (2-4). Within the actinomycetes, members of the genus Streptomyces account for $70-80 \%$ of secondary metabolites (5). However, the rate of discovery of new compounds from terrestrial actinomycetes has decreased. Thus, it is crucial that new groups of actinomycetes from under-exploited environments be pursued as sources of novel bioactive compounds. In recent years, marine environments have become prime resources in the search for and discovery of novel natural products, and marine actinomycetes are considered to be important contributors. Although the exploitation of marine actinomycetes as a source for discovery of bioactive products is at an early stage, numerous metabolites have been isolated in the past few years. New cytotoxic compounds from marine actinomycetes in particular have been reported and these compounds showed significant activity against different cancer cell lines (6-8).

Apoptosis (programmed cell death), which plays a pivotal role in the normal development and pathology of a wide variety of tissues, is characterized by cytoplasmic shrinkage and nuclear condensation $(9,10)$. In recent studies, apoptosis has come to be appreciated as an ideal way to eliminate precancerous and/or cancer cells $(11,12)$. However, most cancer cells block apoptosis which allows malignant cells to survive despite genetic and morphologic transformations. Therefore, searching for agents that trigger apoptosis of tumor cells has become an attractive strategy in anti-cancer drug discovery. Many agents that induce apoptosis are often found to target the mitochondria and promote the activation of caspases, a family of cysteine 
acid proteases, which are important proteolytic enzymes responsible for the execution of apoptosis (13). Once activated, caspases initiate cleavage of a downstream substrate, such as nuclear poly(ADP-ribose) polymerase (PARP), which facilitates cellular disassembly and serves as a marker of cells undergoing apoptosis $(10,14,15)$. Caspases are often regulated by various cellular proteins, including the Bcl-2 family, which promotes (Bax and $\mathrm{Bid}$ ) or inhibit (Bcl-2 and $\mathrm{Bcl}-\mathrm{xL}$ ) apoptosis $(16,17)$. Bcl-2 preserves the integrity of the outer mitochondrial membrane and thereby prevents the release of pro-apoptotic factors from mitochondria $(10,18)$. Although the effects of Bcl-2 on pro-apoptotic factors release are well described, other functions of Bcl-2 in the upstream and downstream regulation of apoptosis remain poorly understood.

In previous years, Akt, a major downstream target of phosphatidylinositol 3-kinase (PI3K), has been linked, through both indirect and direct mechanisms, to a wide variety of antiapoptotic functions $(19,20)$. The activation of Akt signaling, in particular, promotes cell growth and survival. Several studies have shown that PI3K/Akt signaling pathway components are frequently altered in human cancers and overexpression of Akt induces malignant transformation and chemoresistance. Furthermore, combining inhibition of the PI3K/Akt pathway with inhibitors of different signaling pathways may provide a more effective strategy for cancer cells by lowering the threshold for mitochondrial damage and apoptosis $(19,20,21)$. Thus, the Akt pathway presents an exciting new target for molecular therapeutics.

In the course of our screening program of bioactive marine natural products, we isolated 23 marine actinomycetes from seawater samples obtained from Jinhae Bay (southern coast of South Korea). Of these, strain SY-103 exhibited the strongest cytotoxic activity on human leukemic cell lines. Biochemical tests and near-complete $16 \mathrm{~S}$ rDNA analysis indicated that the strain belongs to the genus Streptomyces. Bioassay-guided fractionation by solvent partitioning and purification with reverse-phase high-performance liquid chromatography (HPLC) yielded a pure cytotoxic compound (PCC). In addition, we investigated the effect of PCC on the growth of human leukemia cells (U937, HL-60, K562 and THP-1) and the underlying intracellular signal transduction pathways involved in regulating apoptosis. We found that $\mathrm{PCC}$-induced apoptosis is accompanied by the modulation of Bcl-2 family and activation of caspase-3. It is also suggested that PCC triggers apoptosis through the blockage of the PI3K/Akt signal pathway in leukemia cells.

\section{Materials and methods}

Isolation and identification of cytotoxic marine actinomycetes. Seawater samples were collected from the surface of Jinhae Bay (southern coast of South Korea). Sampling was performed using a Van Dorn sampler in mid-September, 2005. Seawater samples $(0.1 \mathrm{ml})$ were spread on the entire surface of a modified Bennett's agar (22) with aged seawater. After incubation at $25^{\circ} \mathrm{C}$ for 14 days, all colonies with different pigmentation and morphology were chosen for isolation. Among the 23 actinomycete strains isolated, we selected the SY-103 strain that showed the strongest cytotoxic effects on human leukemia cells by MTT [3-(4,5-dimethylthiazol-2-yl)-2,5-diphenyl-tetrazolium bromide, Sigma Chemical Co., St. Louis, MO] assay, which is based on the conversion of MTT to MTT-formazan by mitochondrial enzyme. The strain SY-103 was grown at $25^{\circ} \mathrm{C}$ for seven days in a modified Bennett's medium with shaking. Standard morphological, physiological and biochemical properties were tested according to the methods of Williams et al (23). All carbon-sources for carbon-utilization tests were filter-sterilized and tested at the concentrations recommended by Shirling and Gottlieb (24) and Williams et al (23). Extraction of genomic DNA and 16S rRNA gene amplification were carried out according to the method of Rainey et al (25). The resulting PCR product was ligated into a pGEM-T easy vector (Promega, Madison, WI), sequenced using a Termination Sequencing Ready Reaction kit (Perkin Elmer, Waltham, MA) and analyzed using an ABI 377 genetic analyzer (Perkin Elmer). The near-complete $16 \mathrm{~S}$ rDNA sequence $(1,449 \mathrm{bp})$ was aligned using Clustal W software Ver. 1.7 (26). 16S rDNA sequences used for the phylogenetic analyses were derived and compared with those of other bacterial 16S rDNA sequences available in the DDBJ/EMBL/ GenBank database. Kimura's two-parameter model (27) was applied for the calculation of evolutionary distance. A phylogenetic tree was constructed by the neighbor-joining method (28). Bootstrap analyses of 1,000 replicates were carried out using MEGA version 2.0 (29). Our sequencing and comparison of the 16S rDNA gene of this strain indicated that it was most closely related to Streptomyces californicus NBRC 3386 (99.6\% homology). The 16S rDNA sequence of strain SY-103 has been deposited in the GenBank database under accession number EU643518.

Bacterial culture and isolation of a pure cytotoxic compound. For the production of secondary metabolites, strain SY-103 was cultured, as mentioned above, in ten 5-1 Erlenmeyer flasks containing 31 modified Bennett's medium in each. Flasks were

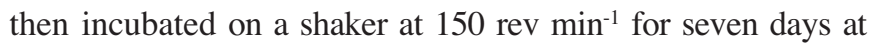
$25^{\circ} \mathrm{C}$. The culture broth was centrifuged $(5,000 \mathrm{x}$ g for $30 \mathrm{~min}$ at $\left.4^{\circ} \mathrm{C}\right)$ to remove cells, and then filtered $(0.2 \mu \mathrm{m}$ pore-size membrane filter) to obtain a cell-free supernatant, followed by partitioning with ethyl acetate (EtOAc) (10 1 x3). The EtOAc layer was concentrated by a rotary vacuum evaporator (Eyela, Japan). The residue $(6.4 \mathrm{~g})$ was subjected to octadesylsilane (ODS) open column chromatography (YMC-GEL, $10 \times 25 \mathrm{~cm}$ ) and eluted with a solvent system from $100 \%$ water to $100 \% \mathrm{MeOH}$ with a stepwise increase of $\mathrm{MeOH}$ concentration by $10 \%$. The $100 \% \mathrm{MeOH}$ fraction $(313 \mathrm{mg}$ ) was further purified by reverse-phase HPLC (Shimadzu, Japan; Cosmosil 5C18-MS column, 10x250 mm; linear gradient of acetonitrile in $\mathrm{H}_{2} \mathrm{O}$ containing $0.05 \%$ trifluoroacetic acid (TFA),

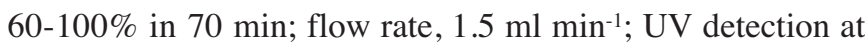
$210 \mathrm{~nm}$ ) to yield a pure compound. The cytotoxic effect of the PCC purified by reverse-phase HPLC on human leukemia cells was measured by MTT assay. PCC was dissolved in methanol as a stock solution at $10 \mathrm{mg} / \mathrm{ml}$ concentration, and stored in aliquots at $-20^{\circ} \mathrm{C}$ until used.

Cell culture and viability assay. Human leukemia cells (HL60, K562, U937 and THP-1) were purchased from the American Type Culture Collection (Rockville, MD). They were cultured in RPMI1640 containing 100 units/ml penicillin/streptomycin 
and supplemented with $10 \%$ heat-inactivated fetal bovine serum (FBS, Gibco BRL, Gaithersburg, $\mathrm{MD}$ ) at $37^{\circ} \mathrm{C}$ and $5 \% \mathrm{CO}_{2}$. The Bcl-2 overexpressing U937 cells were a generous gift from Dr T.K. Kwon (Department of Immunology, Keimyung University School of Medicine, Taegu, Korea) and were maintained in a medium containing $0.7 \mu \mathrm{g} / \mathrm{ml}$ geneticin (G418 sulfate). Measurement of cell viability was determined using the MTT.

Nuclear staining with DAPI. The cells were washed with phosphate-buffered saline (PBS) and fixed with $3.7 \%$ paraformaldehyde in PBS for $10 \mathrm{~min}$ at room temperature. The fixed cells were washed with PBS, and stained with a 4,6diamidino-2-phenylindole (DAPI, Sigma) solution for $10 \mathrm{~min}$ at room temperature. Cells were then washed twice with PBS and analyzed by fluorescence microscopy (Carl Zeiss, Germany).

Agarose gel electrophoresis for DNA fragmentation assay. After the PCC treatment, the cells were lysed in a buffer containing $10 \mathrm{mM}$ Tris- $\mathrm{HCl} \mathrm{pH} 7.4,150 \mathrm{mM} \mathrm{NaCl}, 5 \mathrm{mM}$ EDTA and $0.5 \%$ Triton X-100 for 30 min on ice. The lysates were vortexed and cleared by centrifugation at $10,000 \mathrm{x} g$ for $20 \mathrm{~min}$. The DNA in the supernatant was extracted using a 25:24:1 (v/v/v) equal volume of neutral phenol:chloroform: isoamyl alcohol (Sigma) and analyzed electrophoretically on $1.2 \%$ agarose gels containing $0.1 \mu \mathrm{g} / \mathrm{ml}$ ethidium bromide (30; EtBr, Sigma).

Flow cytometry analysis for measurement of sub-G1 phase. Cells were harvested and washed once with cold PBS, fixed in ice-cold $70 \%$ ethanol and stored at $4^{\circ} \mathrm{C}$. Prior to analysis, the cells were washed once again with PBS, suspended in $1 \mathrm{ml}$ of a cold propidium iodide (PI, Sigma) solution containing $100 \mu \mathrm{g} / \mathrm{ml} \mathrm{RNase} \mathrm{A}, 50 \mu \mathrm{g} / \mathrm{ml} \mathrm{PI}, 0.1 \%$ (w/v) sodium citrate, and $0.1 \%(\mathrm{v} / \mathrm{v}) \mathrm{NP}-40$, and further incubated on ice for $30 \mathrm{~min}$ in the dark. Flow cytometric analyses were carried out using a flow cytometer (FACS Calibur, Becton Dickinson, San Jose, CA) and CellQuest software was used to determine the relative DNA content based on the presence of a red fluorescence (31).

Gel electrophoresis and Western blot analysis. The cells were harvested, lysed, and the protein concentrations were quantified using a BioRad protein assay (BioRad Lab, Hercules, CA), according to the procedure reported by the manufacturer. For Western blot analysis, an equal amount of protein was subjected to electrophoresis on SDS-polyacrylamide gel and transferred to a nitrocellulose membrane (Schleicher \& Schuell, Keene, $\mathrm{NH})$ by electroblotting. The blots were probed with the desired antibodies for $1 \mathrm{~h}$, incubated with the diluted enzyme-linked secondary antibody and visualized by enhanced chemiluminescence (ECL) according to the recommended procedure (Amersham, Arlington Heights, IL). The primary antibodies were purchased from Santa Cruz Biotechnology Inc. (Santa Cruz, CA) and Calbiochem (Cambridge, MA). The peroxidaselabeled donkey anti-rabbit immunoglobulin and peroxidaselabeled sheep anti-mouse immunoglobulin were purchased from Amersham (32).

Caspase-3 activity assay. The enzymatic activity of caspase-3 induced by PCC was assayed using a colorimetric assay kit according to the manufacturer's protocol (R\&D Systems, Minneapolis, MN). Briefly, cells were lysed in a lysis buffer for $30 \mathrm{~min}$ on an ice bath. Lysed cells were centrifuged at $12,000 \mathrm{x} \mathrm{g}$ for $10 \mathrm{~min}$, and $100 \mu \mathrm{g}$ of the protein was incubated with $50 \mu 1$ of a reaction buffer and $5 \mu 1$ of the colorimetric tetrapeptides, Asp-Glu-Val-Asp (DEVD)-p-nitroaniline (pNA) at $37^{\circ} \mathrm{C}$ for $2 \mathrm{~h}$. The optical density of the reaction mixture was quantified spectrophotometrically at a wavelength of $405 \mathrm{~nm}$.

Statistical analysis. The data are expressed as a mean \pm SD. A statistical comparison was performed using one-way ANOVA followed by a Fisher test. The significant differences between the groups were determined using an unpaired Student's t-test. A p-value $<0.05$ was considered significant.

\section{Results}

Isolation and identification of cytotoxic marine actinomycetes. In this study, a total of 23 actinomycete strains were isolated from seawater samples. Cytotoxic effects of metabolites in these 23 strains were tested using the MTT assay, and the result showed that the culture extracts of three strains had strong cytotoxic effects on human leukemia cells (data not shown). Of these, strain SY-103 appeared to have the strongest cytotoxic effects on human leukemia cells such as HL60, K562, U937 and THP-1. This strain was subjected to standard morphological, physiological, and biochemical tests. It was a Gram-positive, aerobic, non-motile actinomycete which formed well-developed and branching substrate mycelium and aerial mycelium. The spores are spherical $(1.2 \mu \mathrm{m}$ in diameter) with a smooth surface. Growth was observed at $10-45^{\circ} \mathrm{C}$, but not at $4^{\circ} \mathrm{C}$. The optimal temperature for growth is $20-30^{\circ} \mathrm{C}$. This strain is tolerant of $\mathrm{NaCl}$ concentrations up to $10 \%$, but not $13 \%$. Nitrate reduction and $\mathrm{H}_{2} \mathrm{~S}$ production are positive. As the sole carbon source, it utilizes L-arabinose, D-fructose, D-mannitol and D-xylose for growth, but not D-arabinose, L-rhamnose or D-sucrose. The above results support the identification of this strain as a member of the genus Streptomyces. Furthermore, a BLAST search of available data in the DDBJ/EMBL/ GenBank database showed the highest similarity (99.6\%) with S. californicus NBRC 3386 (Fig. 1). Thus, we designated this strain to be Streptomyces sp. SY-103. Further taxonomic study will be undertaken for the final identification of this strain.

Isolation of a PCC from a cell-free supernatant of Streptomyces $s p$. SY-103. Bioassay-guided purification of the cytotoxic compound was conducted by determining the cytotoxic activity on human leukemia cells. As above, Streptomyces sp. SY-103 was cultured in ten 5-1 Erlenmeyer flasks containing 31 modified Bennett's medium in each, which were prepared with aged seawater. The flasks were then incubated on a shaker at $150 \mathrm{rev} \mathrm{min}^{-1}$ for seven days at $25^{\circ} \mathrm{C}$. After seven days, the culture broth was centrifuged and filtrated to obtain a cellfree supernatant. The cytotoxic compound was found in the culture broth, but not in the cells. The ethyl acetate layer was concentrated and the residual aqueous suspension $(6.4 \mathrm{~g})$ was subjected to ODS open column chromatography with aqueous $\mathrm{MeOH}$ followed by dichloromethane. The active $100 \% \mathrm{MeOH}$ fraction (313 mg) was further purified by reverse-phase HPLC to yield $53.1 \mathrm{mg}$ of PCC (as described in 


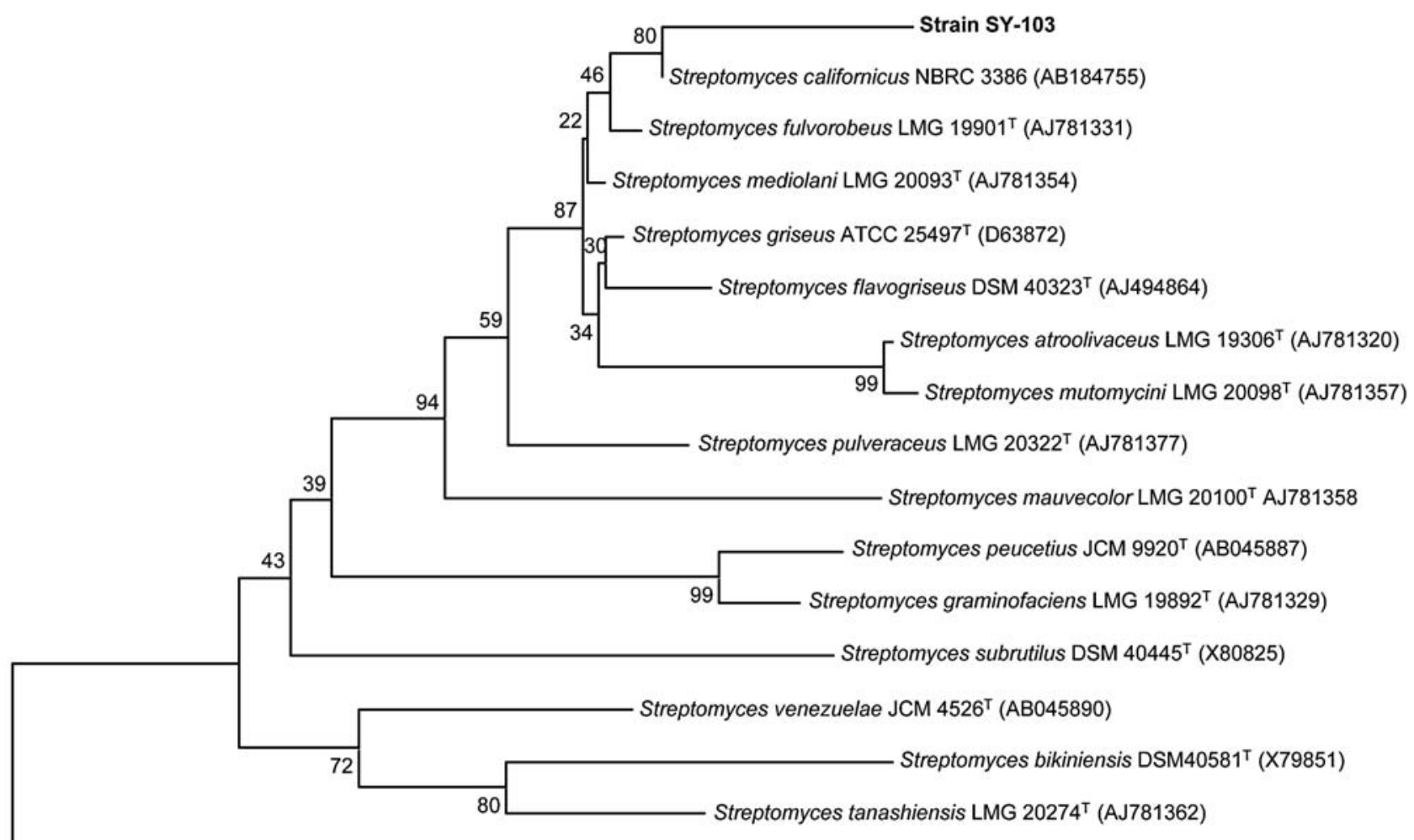

Streptomyces phaeochromogenes DSM 40073 (AF500071)

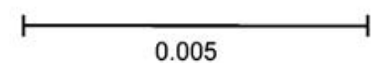

Figure 1. Phylogenetic tree based on 16S rRNA gene sequence comparison indicating the position of Streptomyces sp. SY-103. The phylogenetic tree was generated using the neighbor-joining method. Bootstrap values, expressed as percentages of 1,000 replications, are given at branching points. Bar, 5 nucleotides substitutions per 1,000 nucleotides.

Materials and methods) as a slightly reddish powder, soluble in non-polar solvent. The cytotoxic activity of PCC was examined by MTT assay. Two-dimensional photodiode array detection showed that PCC was a single compound (data not shown).

Inhibition of viability by PCC in leukemia cells. To determine if PCC decreases cell viability, leukemia cells (HL60, K562, U937 and THP-1) were stimulated with $1-50 \mathrm{ng} / \mathrm{ml}$ concentrations of PCC for 6-48 h, and cell viability was measured by an MTT assay. As shown in Fig. 2, the PCC treatment significantly inhibited the cell viability in a concentration- and time-dependent manner. After a 48-h treatment, at $30-50 \mathrm{ng} / \mathrm{ml}$, PCC inhibited the cell viability by $\sim 20 \%$ compared with the controls. However, normal cells grown under the same conditions did not show any toxic effect (data not shown).

Induction of apoptosis by PCC in leukemia cells. Further experiments using fluorescence microscopy, agarose gel electrophoresis, and flow cytometry analyses were carried out to determine if the inhibitory effect of PCC on cell viability is the result of apoptotic cell death. Morphological analysis with DAPI staining showed nuclei with chromatin condensation and the formation of apoptotic bodies in cells cultured with PCC. On the other hand, very few were observed in the control culture (Fig. 3A). Agarose gel electrophoresis indicated that $\mathrm{PCC}$ treatment induced progressive accumulation of fragmented
A)

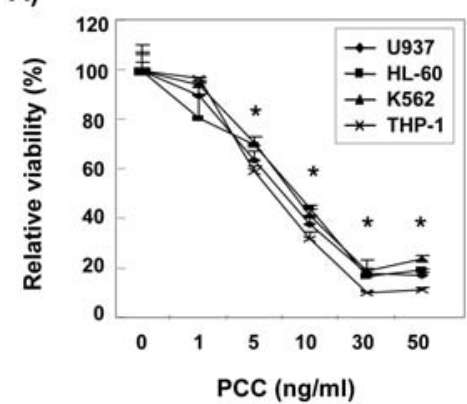

B)

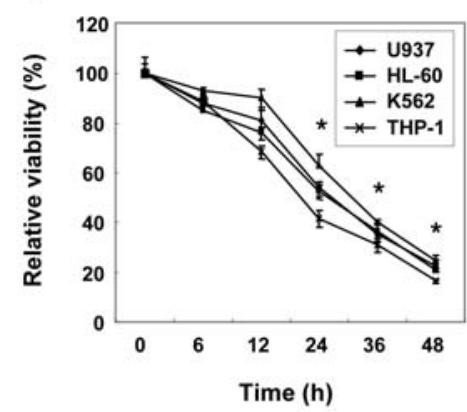

Figure 2. PCC inhibits cell viability in human leukemic cells. The cells were seeded at $5 \times 10^{5}$ cells $/ \mathrm{ml}$ and then treated with the indicated concentrations of PCC for $48 \mathrm{~h}$ (A) or $30 \mathrm{ng} / \mathrm{ml}$ PCC for the indicated times (B). Cell viability was determined by MTT assay. Data are expressed as mean \pm SD of three independent experiments. The significance was determined by Student's t-test ( ${ }^{*} \mathrm{p}<0.05$ vs. untreated control). 
A)

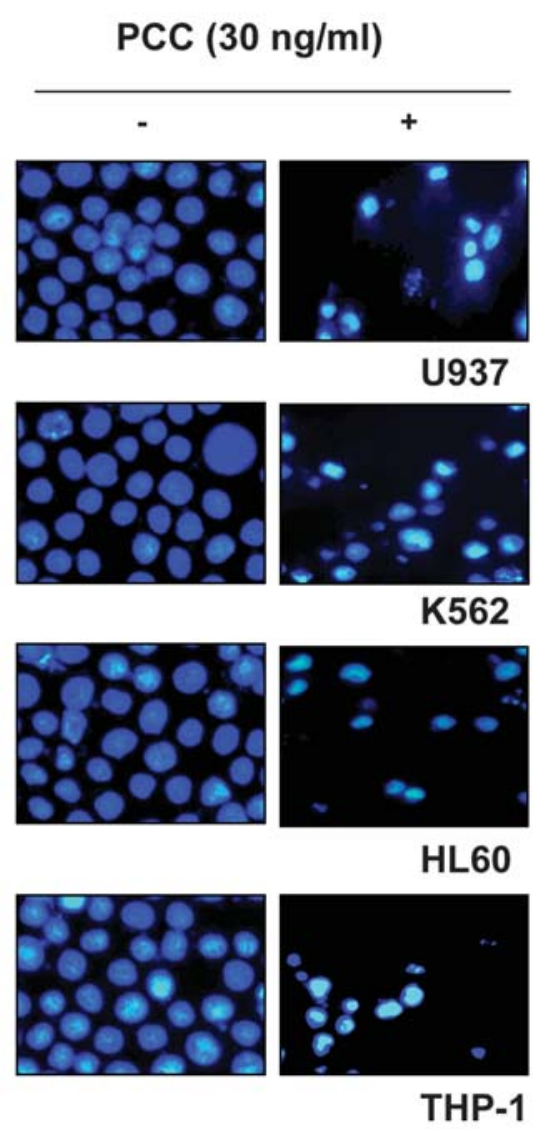

B)

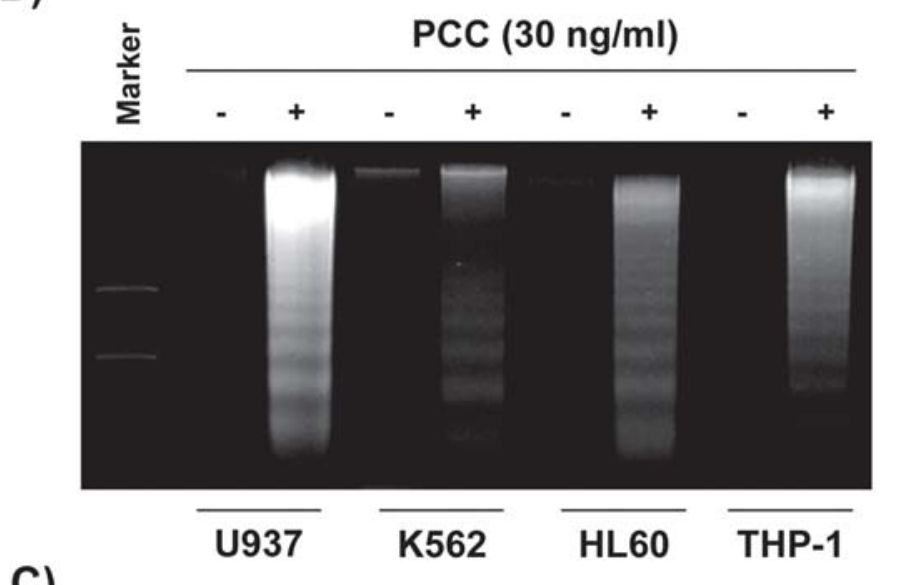

C)

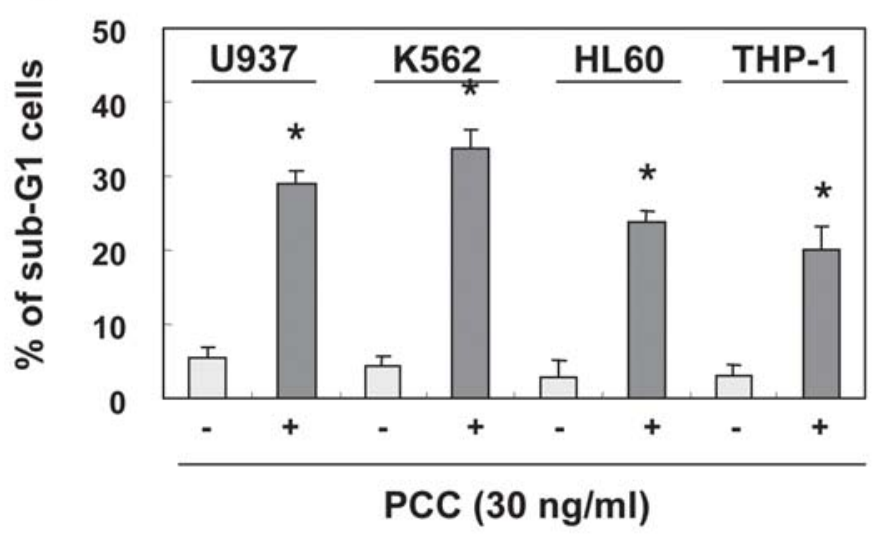

Figure 3. PCC induces apoptosis in human leukemic cells. (A) Cells were treated with $30 \mathrm{ng} / \mathrm{ml}$ of PCC for 48 h, fixed and stained with DAPI. The nuclear morphology was then photographed under fluorescence using a blue filter; magnification was x400. (B) For the analysis of DNA fragmentation, cells were treated with $30 \mathrm{ng} / \mathrm{ml}$ of PCC for $48 \mathrm{~h}$. Genomic DNA was extracted and analyzed on a 1.2\% agarose gel. Marker indicates a size marker of the DNA ladder. (C) To quantify the degree of apoptosis induced by PCC, the cells were evaluated for sub-G1 DNA content using a flow cytometer. Data are expressed as mean $\pm \mathrm{SD}$ of three independent experiments. For statistical analysis, t-test was performed $\left({ }^{*} \mathrm{p}<0.05\right)$.

DNA, which appeared as a typical ladder pattern of DNA fragmentation due to internucleosomal cleavage associated with apoptosis (Fig. 3B). Therefore, flow cytometry analysis was used to determine the magnitude of apoptosis elicited by PCC. As shown in Fig. 3C, addition of PCC resulted in increased accumulation of cells in the sub-G1 phase, which is similar to the results observed when the PCC-induced loss of cell viability, formation of apoptotic bodies, and accumulation of extra-nuclear fragmented DNA was evaluated. This suggests that leukemia cells undergo apoptosis after exposure to PCC, and indicates that there is a good correlation between the extent of apoptosis and the inhibition of cell viability.

Down-regulation of the levels of Bcl-2 expression and activation of caspase- 3 by PCC. Firstly, the apoptotic cascades in leukemia cells caused by PCC were examined by exposing cells to PCC and comparing the levels of Bcl-2 family members. Western immunoblotting showed that the levels of pro-apoptotic Bax expression were not induced in the PCCtreated cells, whereas the level of anti-apoptotic Bcl-2 expression was down-regulated in response to the PCC treatment (Fig. 4A). To determine if PCC-induced apoptosis is associated with the activation of caspases, the expression and activity of caspase- 3 in the PCC-treated leukemia cells were examined by Western blot analysis and an in vitro activity assay. As shown in Fig. 4B, the PCC treatment increased the expression levels of the active subunits and the in vitro activity of caspase-3. This suggests that the activation of caspase- 3 through Bcl-2 down-regulation is a key step in the PCCinduced apoptotic pathway in leukemia cells.

Inhibition of PCC-induced apoptosis by overexpression of $\mathrm{Bcl}-2$. Since Bcl-2 is a well-known anti-apoptotic protein and PCC partly down-regulated the Bcl-2 expression level, the effect of high intracellular levels of $\mathrm{Bcl}-2$ on $\mathrm{PCC}$-induced apoptosis was evaluated using U937/vector and U937/Bcl-2 cells that constitutively express high levels of Bcl-2. The Bcl-2 expression level in U937/Bcl-2 cells was approximately four times higher than in the Bcl-2 in U937/vector cells. As expected, Bcl-2 overexpression significantly blocked the activation of capase-3 induced by PCC, which is consistent with decreased apoptosis in the PCC-treated U937/Bcl-2 cells (Fig. 5A-C). The data demonstrate that $\mathrm{Bcl}-2$ overexpression effectively attenuates PCC-induced apoptosis by blocking the activation of caspase-3.

Inhibition of PCC-induced apoptosis by caspase-3 inhibitor. To show that the activation of caspase- 3 is a key step in the PCC-induced apoptotic pathway, U937 cells were pretreated with z-DEVD-fmk $(50 \mu \mathrm{M})$, a cell-permeable caspase-3 
A)
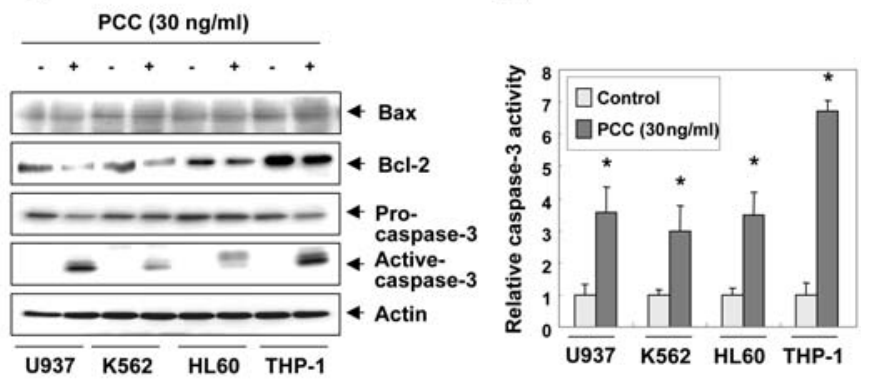

Figure 4. Effects of PCC on the expression of the Bcl-2 family and activation of caspase-3 in human leukemic cells. (A) Cells were treated with $30 \mathrm{ng} / \mathrm{ml}$ of PCC for $48 \mathrm{~h}$. Equal amounts of cell lysates were resolved by SDSpolyacrylamide gels, transferred to nitrocelluloses, and probed with anti-Bax, anti-Bcl-2, and anti-caspase-3 antibodies. Proteins were then visualized using an ECL detection system. Actin was used as the internal control. A representative study is shown, and two additional experiments yielded similar results. (B) The cell lysates from the cells grown under the same conditions as (A) were assayed for in vitro caspase-3 using DEVD-pNA as a substrate. The released fluorescent products were measured as described in Materials and method. Each point represents the mean \pm SD of three independent experiments. The significance was determined by Student's t-test ( ${ }^{*} \mathrm{p}<0.05$ vs. untreated control).

inhibitor, for $1 \mathrm{~h}$, followed by a treatment with $30 \mathrm{ng} / \mathrm{ml}$ PCC for $48 \mathrm{~h}$. The blocking of the caspase-3 activity by pretreatment of cells with z-DEVD-fmk prevented the PCC-induced caspase- 3 activation, chromatin condensation, growth inhibition, and the increase in the sub-G1 population (Fig. 6). These results clearly show that $\mathrm{PCC}$-induced apoptosis is associated with the activation of caspase- 3 .

Blockage of the Akt pathway increases apoptosis by PCC. To investigate the significance of the PI3K/Akt pathway in response to $\mathrm{PCC}$-induced apoptosis, we performed timedependent experiments to determine the expression and phosphorylation levels of Akt. As shown in Fig. 7A, the levels of the total Akt protein remained unchanged during PCCinduced apoptosis; however, its phosphorylation levels were markedly decreased in a time-dependent manner. Next, to evaluate the relationship between PI3K/Akt activity and apoptosis, we investigated whether PCC significantly induces apoptosis in the presence of LY290042 (a specific inhibitor of PI3K/Akt). As shown in Fig. 7B and C, treatment with LY294002 resulted in a marked increase in apoptotic body formation and a decrease in cell viability (from $29 \pm 4$ to $50 \pm 1 \%$ ) in the presence of PCC. We also found that co-treatment of LY294002 with PCC resulted in a significant decrease in cell number (data not shown). Taken together, these indicate that PCC-induced apoptosis in leukemia cells is associated with down-regulation of the PI3K/Akt signaling pathway.

\section{Discussion}

As a promising source for new natural products that have not been found in terrestrial actinomycetes, marine actinomycetes are being investigated for the discovery of new bioactive compounds, with growing and intense interest. These marine actinomycetes are gaining importance not only for their taxonomic and ecological perspectives, but also for their unique metabolites. In particular, most Streptomyces sp. are widely
A)

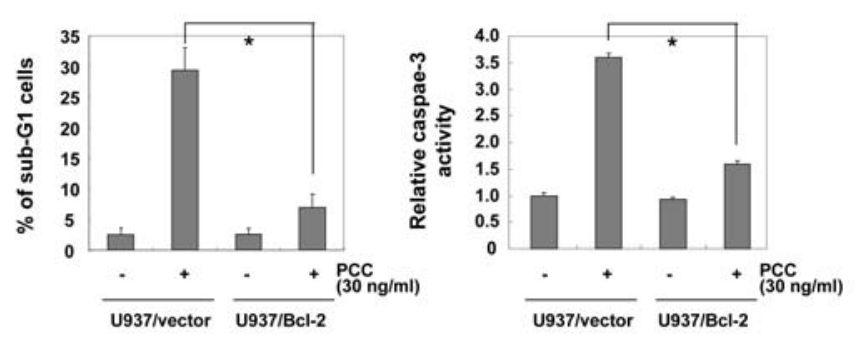

C)

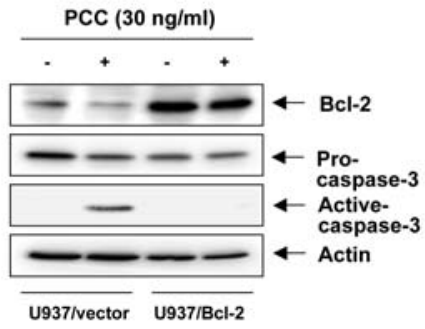

Figure 5. Effects of Bcl-2 overexpression on the apoptosis induction and caspase-3 activation by PCC in U937 cells. (A) U937/vector or U937/Bcl-2 cells were treated with $30 \mathrm{ng} / \mathrm{ml}$ PCC for $48 \mathrm{~h}$, and evaluated for sub-G1 DNA content using a flow cytometer. Data are reported as mean $\pm \mathrm{SD}$ of three independent experiments. (B) After incubation with PCC for $48 \mathrm{~h}$, cell lysates were made and then used to measure caspase- 3 activity using DEVD-pNA, a caspase-3 substrate. Data are reported as mean \pm SD of three independent experiments. (C) Equal amounts of proteins extracted from cells grown under the same conditions as (A) were separated by SDS-polyacrylamide gels and transferred onto nitrocellulose membranes. Membranes were probed with the anti-Bcl-2 and anti-caspase- 3 antibodies. Proteins were visualized using an ECL detection system. Actin was used as the internal control.

distributed elsewhere in the ocean. Therefore, marine Streptomyces species have come to play an increasingly important role in the discovery of new drugs. However, their biotechnological potential has yet to be fully explored. Hence, screening new marine Streptomyces species from oceans that possess useful bioactivities is advantageous in this field.

In the present work, we found that three marine actinomycete strains have strong cytotoxic effects on human leukemia cells. This work shows that marine actinomycetes is a promising source of potential anti-tumor bioactive agents. Of the three strongly cytotoxic strains, strain SY-103 had the strongest cytotoxic effects and was chosen for further study. Biochemical tests and 16S rDNA sequencing of this strain allowed us to identify SY-103 as a strain of genus Streptomyces. Thus, we designated this strain to be Streptomyces sp. SY-103. This strain proved to produce a secondary metabolite that has cytotoxic effects on U937, HL-60, K562, and THP-1 cells. No cytotoxic activity was detected in the cell culture fraction, while the cell-free supernatant showed strong activity. This result suggests that Streptomyces sp. SY-103 produces dissolved cytotoxic compounds that are released into the growth medium. As a result, we successfully isolated PCC from a cell-free supernatant of Streptomyces sp. SY-103 using the reverse-phase HPLC method. The PCC produced seems to be a good candidate for studying cytotoxic compounds. The characteristics of the active compound, PCC, are not yet known, and work is currently being conducted to elucidate its chemical structure. 
A)

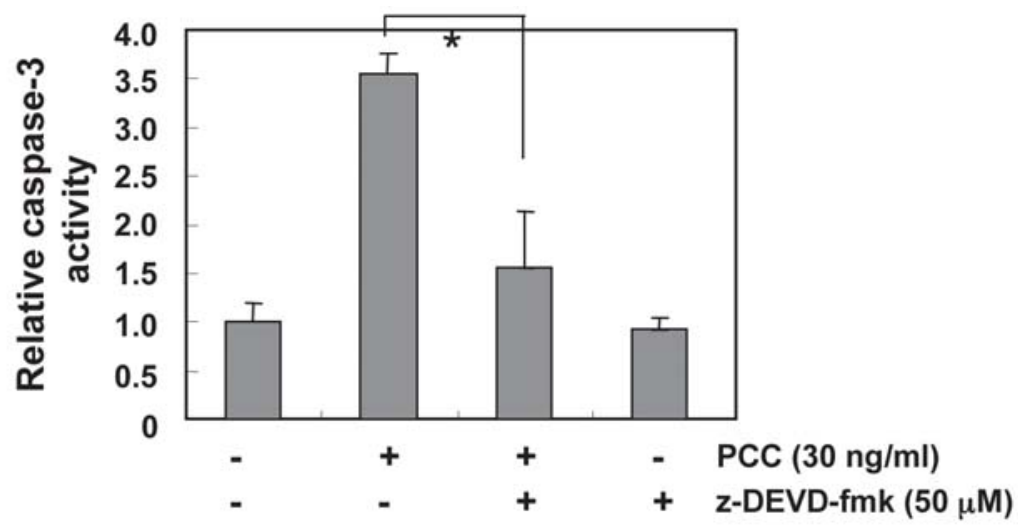

B)
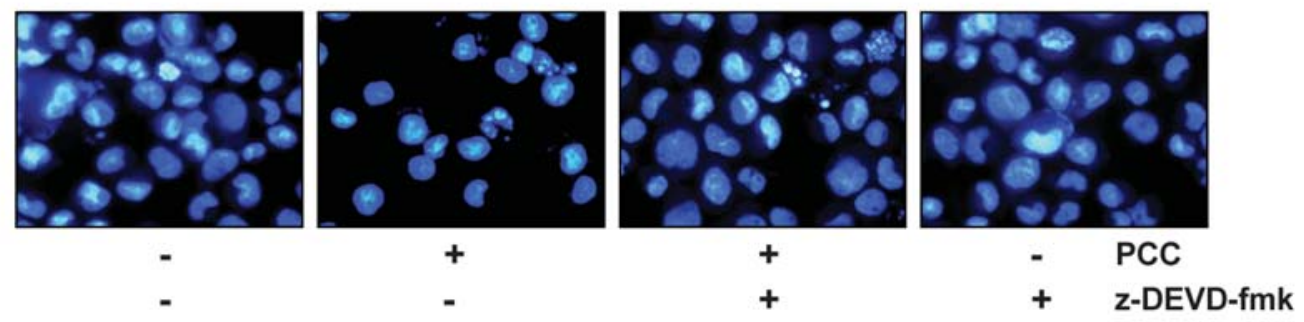

C)

D)
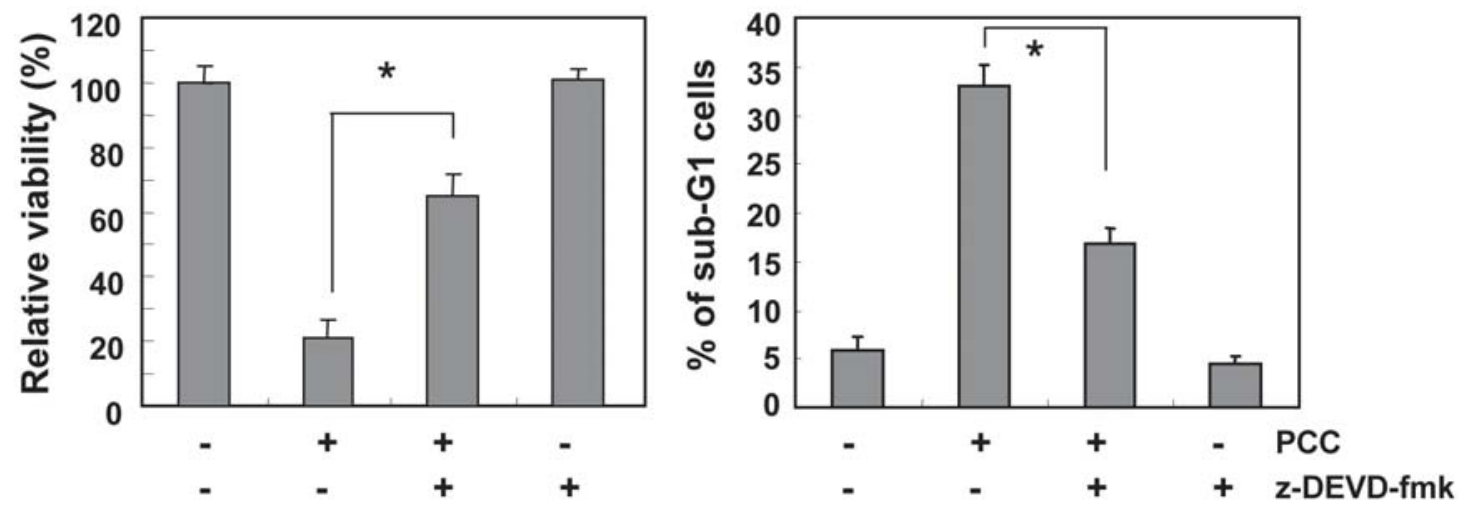

Figure 6. Inhibition of PCC-induced apoptosis by caspase-3 inhibitor in U937 cells. (A) The cells were treated with z-DEVD-fmk (50 $\mu \mathrm{M}$ ) for $1 \mathrm{~h}$ before challenged with $30 \mathrm{ng} / \mathrm{ml}$ PCC for $48 \mathrm{~h}$. Cell lysates were extracted and used to measure caspase-3 activity with DEVD-pNA, a caspase-3 substrate. Data are reported as mean $\pm \mathrm{SD}$ of three independent experiments. (B) Cells grown under the same conditions as (A) were stained with DAPI for 10 min and photographed with a fluorescence microscope using a blue filter; magnification was $\mathrm{x} 400$. (C) Cell viability was determined using MTT assay after $48 \mathrm{~h}$ in the presence of caspase-3 inhibitor, z-DEVD-fmk $(50 \mu \mathrm{M})$ for $1 \mathrm{~h}$ before PCC $(30 \mathrm{ng} / \mathrm{ml})$ treatment. Data are expressed as mean \pm SD of three independent experiments. The significance was determined by Student's t-test ( ${ }^{*} \mathrm{p}<0.05$ vs. untreated control). (D) Cells grown under the same conditions as (A) were evaluated for sub-G1 DNA content using a flow cytometer. Data are reported as mean \pm SD of three independent experiments.

Over the last decade basic cancer research has achieved remarkable advances in cancer biology. However, therapeutic use has been restricted to only a few drugs. To overcome these limitations, it is necessary to isolate new anti-tumor compounds. Recently, apoptosis-inducing compounds purified from Streptomyces strains have been reported. For example, Macherla et al (4) report that three new pyrrolosesquiterpenes isolated from a culture of Streptomyces sp., obtained from an Alaskan marine sediment, exhibited anti-tumor activity toward the colorectal adenocarcinoma HT-29 and melanoma B16-F10 human cancer cell lines. Moreover, Lee et al (33) reported that F3-2-5 isolated from a culture supernatant of Streptomyces sp. induced apoptosis of HeLa cells.

Interest has been focused on the manipulation of the apoptotic process in the treatment of cancer $(9,11,12)$.
Accumulating data indicate that many chemopreventive and/or chemotherapeutic agents cause tumor cell death through the induction of apoptosis. Therefore, the killing of tumors through the induction of apoptosis, which would not cause resistance or side-effects, has now been recognized as a novel strategy for cancer drugs. In the present study, we report that PCC treatment causes a down-regulation of Bcl-2 proteins and activation of caspase-3, and leads to apoptosis in human leukemia cells. Furthermore, Bcl-2 overexpression and caspase-3 inhibitor significantly attenuate PCC-induced apoptosis. In addition, we further examined that the PCC decreased the Akt activation in a time-dependent manner and significantly enhanced apoptosis in pre-treatment LY294002, a specific inhibitor of PI3K.

Mitochondria have been proposed as a novel prospective target for chemotherapy-induced apoptosis $(10,17)$. Since the 
A)

PCC (30 ng/ml)

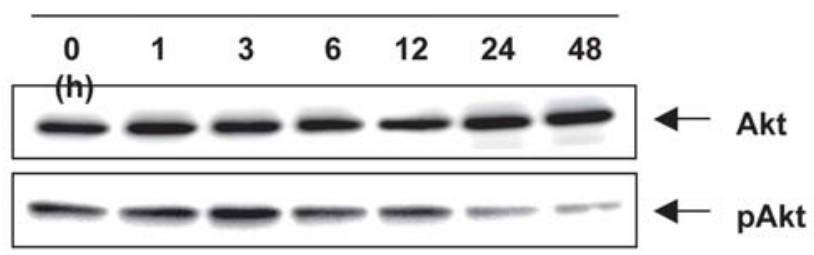

B)
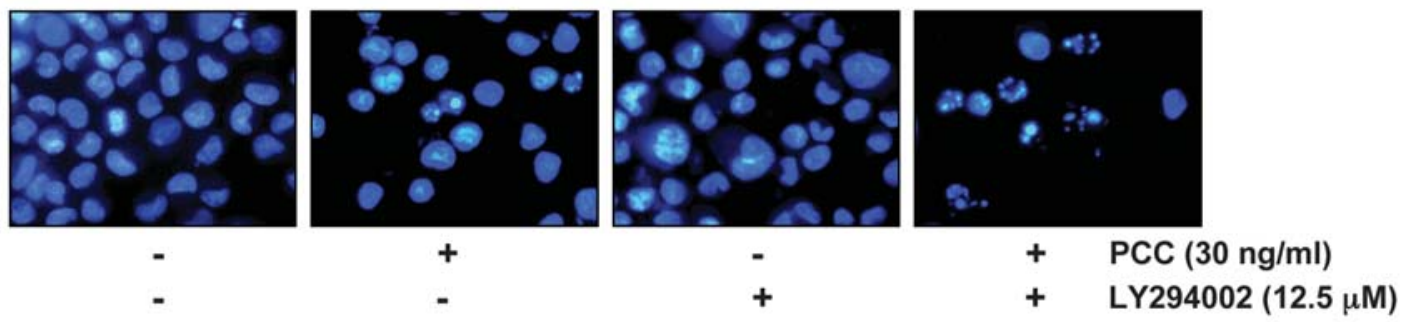

C)

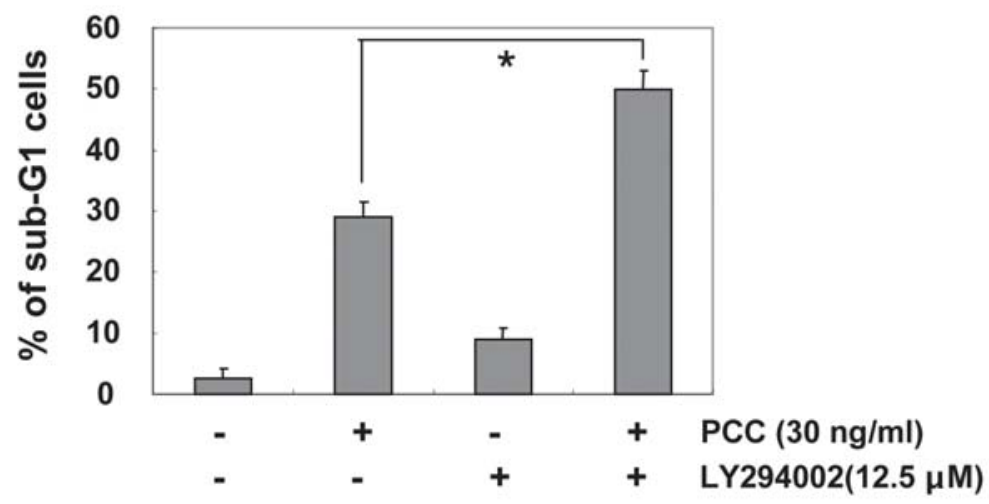

Figure 7. PCC triggers apoptosis through the PI3K/Akt signal in U937 cells. (A) The cells were treated with $30 \mathrm{ng} / \mathrm{ml} \mathrm{PCC}$ for the indicated times. Equal amounts of cell lysate were resolved by SDS-polyacrylamide gels, transferred to nitrocellulose membranes, and probed with the anti-p-Akt and anti-Akt antibodies. Proteins were visualized using an ECL detection system. (B) Cells were stimulated with $30 \mathrm{ng} / \mathrm{ml} \mathrm{PCC}$ for $48 \mathrm{~h}$ after pretreatment of $25 \mu \mathrm{M}$ LY290042 for $1 \mathrm{~h}$. Cells were harvested, fixed and stained with DAPI solution. Stained nuclei were then observed under a fluorescent microscope using a blue filter; magnification was $\mathrm{x} 400$. (C) In a parallel experiment, the degree of apoptosis was determined by a flow cytometer. Each point represents the mean $\pm \mathrm{SD}$ of three independent experiments. The significance was determined by Student's t-test $\left({ }^{*} \mathrm{p}<0.05\right.$ vs. untreated control).

discovery of Bcl-2, several theories have been proposed to unravel the anti-apoptotic properties of this protein $(10,34)$. The anti-apoptotic function of Bcl-2 may be explained by its ability to control several key steps of death signaling. Bcl-2 can form ion channels in biological membranes, and its ion channel activity may control apoptosis by influencing the permeability in the intracellular membranes and releasing them into cytoplasm $(14,15)$. This study demonstrated that PCC treatment results in significant cell growth inhibition and the induction of apoptosis in human leukemia cells and its treatment at a similar dose did not show any toxic effect on normal cells. Our next aim was to identify the mechanism of PCC-induced apoptosis. To test the mechanism of PCCinduced apoptosis, we first determined the effects of PCC on the levels of the Bcl-2 family. Recent studies demonstrate that the Bcl-2 family significantly regulates apoptosis either as an activator (Bax) or as an inhibitor (Bcl-2), and also that the $\mathrm{Bax} / \mathrm{Bcl}-2$ ratio is recognized as a key factor in regulating the apoptotic process $(10,16,17)$. Our data have demonstrated that PCC-induced apoptosis is related to down-regulation of anti-apoptotic Bcl-2 indicating that PCC increases the Bax/Bcl-2 ratio (Fig. 4A) and that induced mitochondrial dysfunction leads to apoptosis of leukemia cells.

The caspase family, aspartate-specific cysteine proteases, also plays a critical role in regulating apoptosis, and the key components of the biochemical pathways of caspase activation have been recently elucidated (13). Caspase signaling is initiated, and then propagated by proteolytic autocatalysis and the cleavage of downstream caspases and substrates. Capases-3 is one of the key executioners of apoptosis, as it is either partially or totally responsible for the proteolytic cleavage of many key proteins, such as PARP (15). PARP is important for cell viability, but the cleavage of PARP facilitates cellular disassembly, and serves as a marker of cells undergoing apoptosis $(14,15)$. In this study, we demonstrated that PCCinduced apoptosis of leukemia cells was associated with activation of caspase-3 (Fig. 4) and, under the same conditions, a specific caspase-3 inhibitor, z-DEVD-fmk, was able to prevent the PCC-induced apoptosis by blocking not only caspase-3 activation, but also apoptotic body formation 
(Fig. 6). Furthermore, it was shown that Bcl-2 overexpression significantly attenuates PCC-induced apoptosis in U937 cells by inhibiting caspase-3 activity (Fig. 5). The transfection data show that PCC-induced apoptosis and caspase- 3 activation are related to the down-regulation of Bcl-2, which suggests that the overexpression of Bcl-2 blocks the activation of caspase- 3 and increases the threshold for PCC-induced cell death. Therefore, the data showing that PCC down-regulates the expression of Bcl-2 in leukemia cells suggest that the downregulation of $\mathrm{Bcl}-2$ contributes to the activation of caspase- 3 in the PCC-induced apoptotic pathway.

$\mathrm{PI} 3 \mathrm{~K} /$ Akt signal pathway plays a critical role in regulating cell survival and death in many physiological and pathological settings. The PI3K/Akt pathway is more often associated with cell survival through activation of anti-apoptotic downstream effectors $(19,20)$. The purpose of the present study was to determine the effects of PI3K/Akt on PCC-induced apoptosis. Our results demonstrated that $\mathrm{PCC}$ induces a down-regulation of the PI3K/Akt signaling pathway, and the inhibition of the PI3K/Akt pathway significantly increased PCC-induced apoptosis (Fig. 7). However, PCC did not affect the activity of mitogen-activated protein kinases (MAPKs), a family of serine/threonine kinases, such as c-Jun $\mathrm{NH}_{2}$-terminal kinase (JNK), p38 MAPK, and extracellular signal-regulated kinase (ERK) $1 / 2$; and blocking the ERK, JNK, and p38 pathways did not alter the apoptosis with treated PCC (data not shown). These data strongly suggest that PCC-induced apoptosis is associated with PI3K/Akt pathway.

In summary, we have demonstrated that apoptosis is significantly induced in human leukemia cells exposed to PCC isolated from Streptomyces sp. SY-103. This apoptotic response was associated with modulation of the Bcl-2 family and caspase- 3 activation. Moreover, the inactivation of PI3K/ Akt pathway may perform important roles in PCC-induced apoptosis. Therefore, it was thought that PCC is a promising molecule for cancer chemoprevention or chemotherapy. Further efforts to identify the structure of PCC and to explore the therapeutic strategy are necessary.

\section{Acknowledgements}

Drs Min Ho Han and Cheng-Yun Jin are recipients of postdoctoral fellowship from the Ministry of Education, Science and Technology through the Brain Korea 21 Project. This research was supported by a grant (M2007-03) from Marine Bioprocess Research Center of the Marine Bio 21 Center funded by the Ministry of Land, Transport and Maritime, Republic of Korea.

\section{References}

1. Berdy J: Bioactive microbial metabolites. J Antibiot 58: 1-26, 2005.

2. Jeong SY, Shin HI, Kim TS, Lee HS, Park SK and Kim HM: Streptokordin, a new cytotoxic compound of the methylpyridine class from a marine-derived Streptomyces sp. KORDI-3238. J Antibiot 59: 234-240, 2006.

3. Liu R, Cui CB, Duan L, Gu QQ and Zhu WM: Potent in vitro anticancer activity of metacycloprodigiosin and undecyprodigiosin from a sponge-derived actinomycete Saccharopolyspora sp. nov. Arc Pharm Res 28: 1341-1344, 2005.

4. Macherla VR, Liu J, Bellows C, Teisan S, Nicholson B, Lam KS and Potts BC: Glaciapyrroles A, B, and C, pyrrolosesquiterpenes from a Streptomyces sp. isolated from an Alaskan marine sediment. J Nat Prod 68: 780-783, 2005.
5. Challis GL and Hopwood DA: Synergy and contingency as driving forces for the evolution of multiple secondary metabolite production by Streptomyces species. Proc Natl Acad Sci USA 100 (suppl 2): 14555-14561, 2003.

6. Maskey RP, Halmke E, Kayser O, Fiebig HH, Maier A, Busche A and Laatsch $\mathrm{H}$ : Anti-cancer and antibacterial trioxacarcins with anti-malaria activity from a marine Streptomyces and their absolute stereochemistry. J Nat Prod 57: 771-779, 2004.

7. Soria-Mercado IE, Prieto-Davo A, Jensen PR and Fenical W: Antibiotic terpenoid chloro-dihydroquinones from a new marine actinomycete. J Nat Prod 68: 904-910, 2005.

8. Stritzke K, Schulz S, Laatsch H, Helmke E and Beil W: Novel caprolactones from a marine Streptomyces. J Nat Prod 67: 395-401, 2004.

9. Hengartner MO: The biochemistry of apoptosis. Nature 407: 770-776, 2000

10. Jeong SY and Seol DW: The role of mitochondria in apoptosis. BMB Rep 41: 11-22, 2008.

11. McConkey DJ: Therapy-induced apoptosis in primary tumors. Adv Exp Med Biol 608: 31-51, 2007.

12. Mow BM, Blajeski AL, Chandra J and Kaufmann SH: Apoptosis and the response to anticancer therapy. Curr Opin Oncol 13: 453-462, 2001

13. Stennicke HR and Salvesen GS: Properties of the caspases. Biochim Biophys Acta 1387: 17-31, 1998.

14. Fernandes-Alnemri T, Litwack G and Alnemri ES: CPP32, a novel human apoptotic protein with homology to Caenorhabditis elegans cell death protein Ced-3 and mammalian interleukin-1 ß-converting enzyme. J Biol Chem 269: 30761-30764, 1994.

15. Oliver FJ, de la Rubia G, Rolli V, Ruiz-Ruiz MC, de Murcia G and Murcia JM: Importance of poly(ADP-ribose) polymerase and its cleavage in apoptosis. Lesson from an uncleavable mutant. J Biol Chem 273: 33533-33539, 1998.

16. Adams JM and Cory S: The Bcl-2 protein family: arbiters of cell survival. Science 281: 1322-1326, 1998.

17. Thees S, Hubbard GB, Winckler J, Schultz C and Rami A Specific alteration of the $\mathrm{Bax} / \mathrm{Bcl} 2$ ratio and cytochrome $\mathrm{c}$ without execution of apoptosis in the hippocampus of aged baboons. Restor Neurol Neurosci 23: 1-9, 2005.

18. Qiao X, Chen X, Wu D, Ding R, Wang J, Hong Q, Shi S, Li J Xie Y, Lu Y and Wang Z: Mitochondrial pathway is responsible for aging-related increase of tubular cell apoptosis in renal ischemia/reperfusion injury. J Gerontol A Biol Sci Med Sci 60: 830-839, 2005.

19. Datta SR, Brunet A and Greenberg ME: Cellular survival: a play in three Akts. Genes Dev 13: 2905-2927, 1999.

20. Nicholson KM and Anderson NG: The protein kinase B/Akt signalling pathway in human malignancy. Cell Signal 14: 381-395, 2002.

21. Kennedy SG, Wagner AJ, Conzen SD, Jordán J, Bellacosa A Tsichlis PN and Hay N: The PI3-kinase/Akt signaling pathway delivers an anti-apoptotic signal. Genes Dev 11: 701-713, 1997.

22. Atlas RM: Handbook of Microbiological Media. Parks LC (ed). CRC Press, Boca Raton, 1993.

23. Williams ST, Goodfellow M and Alderson G: Genus Streptomyces Waksman and Henrici 1943. In: Bergey's Manual of Systematic Bacteriology. Williams ST (ed). Vol. 4 Williams \& Wilkins, Baltimore, pp2452-2492, 1989.

24. Shirling EB and Gottlieb D: Methods for characterization of Streptomyces species. Int J Syst Bacteriol 16: 313-340, 1966.

25. Rainey FA, Ward-Rainey N, Kroppenstedt RM and Stackebrandt E: The genus Norcardiopsis represents a phylogenetically coherent taxon and a distinct actinomycete lineage: proposal of Norcardiopsaceae fam. nov. Int J Syst Bacteriol 46: 1088-1092, 1996.

26. Thompson JD, Higgins DG and Gibson TJ: CLUSTAL W: improving the sensitivity of progressive multiple sequence alignment through sequence weighting, position-specific gap penalties and weight matrix choice. Nucleic Acids Res 22: 4673-4680, 1994.

27. Kimura M: A simple method for estimating evolutionary rates of base substitution through comparative studies of nucleotide sequences. J Mol Evol 16: 111-120, 1980.

28. Saitou $\mathrm{N}$ and Nei M: The neighbor-joining method: a new method for reconstructing phylogenetic trees. Mol Biol Evol 4: 189-204, 1987.

29. Kumar S, Tamura K, Jakobsen IB and Nei M: MEGA2: Molecula evolutionary genetics analysis software. Bioinformatics 17 : 1244-1245, 2001 
30. Lee KB, Lee JS, Park JW, Huh TL and Lee YM: Low energy proton beam induces tumor cell apoptosis through reactive oxygen species and activation of caspases. Exp Mol Med 40: 118-129, 2008

31. Jung EJ, Kim CW and Kim DR: Cytosolic accumulation of gammaH2AX is associated with tropomyosin-related kinase Ainduced cell death in U2OS cells. Exp Mol Med 40: 276-285, 2008.

32. Kim SY, Kim JC, Kim JK, Kim HJ, Lee HM, Choi MS, Maeng PJ and Ahn JK: Hepatitis B virus X protein enhances NF- $\mathrm{B}$ activity through cooperating with VBP1. BMB Rep 41: 158-163, 2008 .
33. Lee CH, Lim H, Moon S, Shin C, Kim S, Kim BJ and Lim Y: Novel anticancer agent, benzyldihydroxyoctenone, isolated from Streptomyces sp. causes G1 cell cycle arrest and induces apoptosis of HeLa cells. Cancer Sci 98: 795-802, 2007.

34. Kluck RM, Bossy-Wetzel E, Green DR and Newmeyer DD: The release of cytochrome $\mathrm{c}$ from mitochondria: a primary site for Bcl-2 regulation of apoptosis. Science 275: 1132-1136, 1997. 